\title{
About the Legendre type operators
}

\author{
Evgeny Maleko* \\ Ural State University of Economics, 620000 Ekaterinburg, Russia
}

\begin{abstract}
The article considers Legendre type operators acting in the corresponding weight separable Hilbert spaces. The choice of these spaces is due to the fact that these operators preserve all properties of the Legendre operator acting on $\mathrm{L}_{2}(-1,1)$. In particular,1) the Legendre type operators, operating in respective weight separable Hilbert spaces, remain discrete,2) the spectrum does not change relative to the classical Legendre operator,3) the corresponding eigenfunctions are compositions of Legendre polynomials and some functions.
\end{abstract}

\section{Introduction}

Retrieval of operators which have known spectral properties in advance is one of the most important problems for contemporary mathematics. If we construct two operators, $\mathbb{P}_{+}(v)$ and $\mathbb{P}_{-}(v)$, in a certain separable Hilbert space (hereinafter referred to as SHS), and one of them will recurrently find certain sequence of functions from SHS, while the other will find the same sequence but in reverse order, though recurrently as well, when we multiply these two operators, we may get explicitly operative operator in SHS having the following peculiarities: the constructed functions turn out to be the eigenfunctions for this operator.

The article also describes the construction of some of the weighted separable Hilbert spaces $L_{2}^{\omega}(a, b), \omega=\omega(x), x \in[a, b]$, denoted by $\mathbb{H}^{\omega, a, b}$. These spaces $\mathbb{H} \omega, \mathrm{a}, \mathrm{b}$ are homeomorphic to the space $\mathbb{H}$. The homeomorphism will be proved by the properties of the operator $\widehat{A}$ hereinafter. We will also study the action of the operator $\widehat{A}$ in $\mathbb{H} \omega, a, b$.

\section{Construction of the Legendre type operator}

Let us assume that in a certain separable Hilbert space $\mathbb{H}=\mathrm{L}_{2}(-1,1)$, the following differential operators are operative:

$$
\begin{aligned}
& \mathbb{P}_{+}(v):=-\frac{f_{1}(x)}{\vartheta+1} \frac{d}{d x}+f_{2}(x) \mathbb{I}, \\
& \mathbb{P}_{-}(v):=-\frac{f_{1}(x)}{\vartheta} \frac{d}{d x}+f_{2}(x) \mathbb{I},
\end{aligned}
$$

where the functions $f_{1}=f_{1}(x)$ and $f_{2}=f_{2}(x)$ meet the following requirements:

1) $f_{2}$ is doubly continuously differentiable function within $[-1,1]$;

2)for any $x \in[-1,1]$ the following equation will be true: $f_{1}(x) f_{2}{ }^{\prime}(x)+f_{2}{ }^{2}(x)=1$;

\footnotetext{
*Corresponding author: emaleko@ rambler.ru
} 
3) $f_{2}(x)$ is a function of fixed signs for any $x \in[-1,1]$.

Operator $\mathbb{I}$ is an identity operator. The definition range for $\mathbb{P}_{+}(v)\left(-1 \neq v \in \mathbb{R}^{1}\right)$ and $\mathbb{P}_{-}(v)$ $\left(0 \neq v \in \mathbb{R}^{1}\right)$ operators includes all the functions which are absolutely continuous together with their first-order derivatives within the range of $[-1,1]$, while second-order derivatives within $[-1,1]$ must be square summable. It is easy to verify that the definition ranges for $\mathbb{P}$. $(v+1) \mathbb{P}_{+}(v)\left(-1 \neq v \in \mathbb{R}^{1}\right)$ and $\mathbb{P}_{+}(v-1) \mathbb{P}_{-}(v)\left(0 \neq v \in \mathbb{R}^{1}\right)$ operators include all the functions which are absolutely continuous together with their first-order derivatives within the range of $[-1,1]$, herewith, within the same range, second-order derivatives must be square summable. In this case, it is obvious that $\mathrm{D}\left(\mathbb{P}_{-}(v+1) \mathbb{P}_{+}(v)\right)=\mathrm{D}_{(}\left(\mathbb{P}_{+}(v-1) \mathbb{P}_{-}(v)\right)$ and $\overline{\mathrm{D}\left(\mathbb{P}_{-}(v+1) \mathbb{P}_{+}(v)\right)}=\overline{\mathrm{D}\left(\mathbb{P}_{+}(v-1) \mathbb{P}_{-}(v)\right)}=\mathbb{H}$, where the line above them means norm closure in $\mathbb{H}$.

Definition 1. Operator $\widehat{A}$ which is operative in $\mathbb{H}$ is such that $D(\widehat{A})=D\left(\mathbb{P}_{-}(v+\right.$ 1) $\left.\mathbb{P}_{+}(v)\right)=\mathrm{D}\left(\mathbb{P}_{+}(v-1) \mathbb{P}_{-}(v)\right)$ and for $D_{v}(\widehat{A}):=D(\widehat{A}) \cap \mathbb{T}_{v} \forall \vartheta \in \mathbb{C}$ the following operator equations are simultaneously true:

$$
\begin{gathered}
(\vartheta+1)^{2}\left(\mathbb{P}_{-}(v+1) \mathbb{P}_{+}(v)-\mathbb{I}\right)=\mathrm{f}_{1} \mathrm{f}_{2}^{\prime}(\widehat{\mathrm{A}}-\vartheta(\vartheta+1) \mathbb{I})=\mathbb{O} \\
\vartheta^{2}\left(\mathbb{P}_{+}(v-1) \mathbb{P}_{-}(v)-\mathbb{I}\right)=\mathrm{f}_{1} \mathrm{f}_{2}^{\prime}(\widehat{A}-\vartheta(\vartheta+1) \mathbb{I})=\mathbb{O}
\end{gathered}
$$

will be called Itextbf $\{$ the Legendre type operator $\}$. Here $\mathbb{O}$ is the annihilator in $\mathbb{H}$,

$$
\mathbb{T}_{\vartheta}:=\left\{g \in \mathrm{D}(\widehat{\mathrm{A}}):(\vartheta+1)^{2}\left(\mathbb{P}_{-}(v+1) \mathbb{P}_{+}(v)-\mathbb{I}\right) \mathrm{g}=0 \wedge \vartheta^{2}\left(\mathbb{P}_{+}(v-1) \mathbb{P}_{-}(v)-\mathbb{I}\right) \mathrm{g}=0\right\}
$$

Let us prove that this definition is correct. At first, let us consider the operator multiplications:

$$
\begin{gathered}
\mathbb{P}_{-}(v+1) \mathbb{P}_{+}(v)=-\frac{f_{1}}{(\vartheta+1)^{2}}\left(f_{1}^{\prime} \frac{d}{d x}+f_{1} \frac{d^{2}}{d x^{2}}\right)+\left(\frac{f_{1} f_{2}^{\prime}}{\vartheta+1}+f_{2}^{2}\right) \mathbb{I} \\
\mathbb{P}_{+}(v-1) \mathbb{P}_{-}(v)=-\frac{f_{1}}{\vartheta^{2}}\left(f_{1}^{\prime} \frac{d}{d x}+f_{1} \frac{d^{2}}{d x^{2}}\right)+\left(-\frac{f_{1} f_{2}^{\prime}}{\vartheta}+f_{2}^{2}\right) \mathbb{I}
\end{gathered}
$$

Given that

$$
\mathbb{P}_{-}(v+1) \mathbb{P}_{+}(v)=\mathbb{I}
$$

and

$$
\mathbb{P}_{+}(v-1) \mathbb{P}_{-}(v)=\mathbb{I}
$$

for $\mathrm{D}_{v}(\widehat{\mathrm{A}})$, we will multiply expression $(3)$ by $(\vartheta+1)^{2}$ :

$$
\begin{gathered}
(\vartheta+1)^{2} \mathbb{P}_{-}(v+1) \mathbb{P}_{+}(v)= \\
-f_{1} f_{1}^{\prime} \frac{d}{d x}-f_{1}^{2} \frac{d^{2}}{d x^{2}}+(v+1) f_{1} f_{2}^{\prime} \mathbb{I}+(\vartheta+1)^{2} f_{2}^{2} \mathbb{I}=(\vartheta+1)^{2} \mathbb{I}
\end{gathered}
$$

and expression (4) we will multiply by $\vartheta^{2}$ :

$$
\vartheta^{2} \mathbb{P}_{+}(v-1) \mathbb{P}_{-}(v)=-f_{1} f_{1}^{\prime} \frac{d}{d x}-f_{1}^{2} \frac{d^{2}}{d x^{2}}+\vartheta f_{1} f_{2}^{\prime} \mathbb{I}+\vartheta^{2} f_{2}^{2} \mathbb{I}=\vartheta^{2} \mathbb{I},
$$

From (5) and (6), for any $\forall \vartheta \in \mathbb{C}$ the following equation will be true:

$$
(2 \vartheta+1)\left(\mathrm{f}_{1} \mathrm{f}_{2}^{\prime}+\mathrm{f}_{2}^{2}-1\right)=0
$$


Based on (7), from (5) and (6) we have as follows:

$$
\begin{gathered}
(\vartheta+1) \mathrm{f}_{1} \mathrm{f}_{2}^{\prime} \mathbb{I}+(\vartheta+1)^{2} \mathrm{f}_{2}^{2} \mathbb{I}-(\vartheta+1)^{2} \mathbb{I}=-\vartheta \mathrm{f}_{1} \mathrm{f}_{2}^{\prime} \mathbb{I}+\vartheta^{2} \mathrm{f}_{2}^{2} \mathbb{I}-\vartheta^{2} \mathbb{I}= \\
\vartheta(\vartheta+1)\left(\mathrm{f}_{2}^{2}-1\right) \mathbb{I}=-\vartheta(\vartheta+1) \mathrm{f}_{1} \mathrm{f}_{2}^{\prime} \mathbb{I} .
\end{gathered}
$$

Taking into account (8), from (5) and (6) we will get:

$$
\begin{gathered}
\mathbb{Q}=(\vartheta+1)^{2}\left(\mathbb{P}_{-}(\vartheta+1) \mathbb{P}_{+}(\vartheta)-\mathbb{I}\right)=-f_{1}\left(f_{1}^{\prime} \frac{d}{d x}+f_{1} \frac{d^{2}}{d x^{2}}+\vartheta(\vartheta+1) f_{2}^{\prime} \mathbb{I}\right), \\
\mathbb{O}=\vartheta^{2}\left(\mathbb{P}_{+}(\vartheta-1) \mathbb{P}_{-}(\vartheta)-\mathbb{I}\right)=-f_{1}\left(f_{1}^{\prime} \frac{d}{d x}+f_{1} \frac{d^{2}}{d x^{2}}+\vartheta(\vartheta+1) f_{2}^{\prime} \mathbb{I}\right) .
\end{gathered}
$$

Right-hand members of the last two operator equations are equal. It is easy to extract the Legendre operator from them:

$$
\widehat{A}=-\frac{f_{1}}{f_{2}^{\prime}} \frac{d^{2}}{d x^{2}}-\frac{f_{1}^{\prime}}{f_{2}^{\prime}} \frac{d}{d x}
$$

thus, the correctness of the above definition is obvious.

\section{Several properties of the operator $\widehat{A}$}

\subsection{Equivalence}

\section{Statement 1.}

For any complex number $\vartheta$, the operator equation

$$
\widehat{A}=\vartheta(\vartheta+1) \mathbb{I}
$$

and the equations set

$$
\left\{\begin{array}{c}
(\vartheta+1)^{2}\left(\mathbb{P}_{-}(\vartheta+1) \mathbb{P}_{+}(\vartheta)-\mathbb{I}\right)=\mathbb{O} \\
\vartheta^{2}\left(\mathbb{P}_{+}(\vartheta-1) \mathbb{P}_{-}(\vartheta)-\mathbb{I}\right)=\mathbb{O}
\end{array}\right.
$$

shall be equivalent within the set of all the functions from $D_{v}(\widehat{A}) \subset \mathbb{H} \forall \vartheta \in \mathbb{C}$.

The above Statement may be proved obviously using (2).

\subsection{Self-adjointness of operator $\widehat{\mathbf{A}}$}

Let's look at the identity:

$$
-\left(f_{1} y\right)^{\prime}+f_{2}^{\prime} y \equiv f_{2}^{\prime}(\widehat{A}+\mathbb{I}) y
$$

and define as follows: $y^{[1]}=f_{1} \frac{d y}{d x}, y^{[2]}=f_{1}^{\prime} y-\frac{d}{d x} y^{[1]}$. In this case, $y^{[1]}$ and $y^{[2]}$ are quasiderivatives which correspond to the differential expression $-\left(f_{1} y\right)^{\prime}+f_{2}^{\prime} y$. In this can we can introduce the following operator $\mathrm{By}:=\mathrm{y}^{[2]}$ whose properties have been well studied in [2], which, in its turn, will allow us to look at the operator $\widehat{A}$ from 'classic' point of view. To do so we will need some data from [2, Chapter 5], [3]. Let us remind them:

a) Quasiderivative functions $\mathrm{y}=\mathrm{y}(\mathrm{x})\left(=\mathrm{y}^{[0]}(\mathrm{x})\right)$, corresponding to the expression below 


$$
1(\mathrm{y}):=(-1)^{\mathrm{n}}\left(\mathrm{p}_{0} \mathrm{y}^{(\mathrm{n})}\right)^{(\mathrm{n})}+(-1)^{\mathrm{n}-1}\left(\mathrm{p}_{1} \mathrm{y}^{(\mathrm{n}-1)}\right)^{(\mathrm{n}-1)}+\ldots+\mathrm{p}_{\mathrm{n}} \mathrm{y}
$$

are the functions $\mathrm{y}^{[1]}, \mathrm{y}^{[2]}, \ldots, \mathrm{y}^{[2 \mathrm{n}]}$ which are defined be the following formulas:

$$
\left\{\begin{array}{c}
y^{[k]}=\frac{d^{k} y}{d x^{k}}, \\
y^{[n]}=p_{0} \frac{d^{n} y}{d x^{n}}, \\
y^{[n+k]}=p_{0} \frac{d^{n-k} y}{d x^{n-k}}-\frac{d}{d x}\left(y^{[n+k-1]}\right), \quad k=1,2, \ldots, n-1,
\end{array}\right.
$$

from this it follows that $1(\mathrm{y})=\mathrm{y}^{[2 \mathrm{n}]}$;

b) $1(y)$ is a self-adjoint differential expression, if its coefficients are real-valued functions differentiable sufficient number of times;

c) If $(a, b)$ is the range where the differential expression $1(y)$ is considered, and functions $1 / \mathrm{p}_{0}(\mathrm{x}), \mathrm{p}_{1}(\mathrm{x}), \ldots, \mathrm{p}_{\mathrm{n}}(\mathrm{x})$ are measurable within $(\mathrm{a}, \mathrm{b})$ and summable in its every isolated finate subinterval $[\alpha, \beta]$, then $1(y)$ is a regular differential expression;

d) If the above item c) will have at least one of the conditions violated, with the conditions being the measurability of functions $\left.1 / \mathrm{p}_{0}(\mathrm{x})\right\}, \mathrm{p}_{1}(\mathrm{x}), \ldots, \mathrm{p}_{\mathrm{n}}(\mathrm{x})$ within the range of $(\mathrm{a}, \mathrm{b})$ and summability within its every finate subinterval $[\alpha, \beta]$, then $1(y)$ is singular differential expression.

In our case, if the function $1 / f_{1}(x)$ is summable within $(-1,1)$, then the operator $B:=f_{2}^{\prime}($ $A \hat{+})\left(:=y^{[2]}\right)$ will be called $\backslash$ textbf $\{$ regular $\}$ (according to Naimark, compare to item c) of the above paragraph); if the summability condition is violated for the function $1 / f_{1}(x)$, it will be a singular operator (compare to item $\mathrm{d}$ ) of the above paragraph).

Statement 2. Operators $\widehat{A}:=\frac{1}{f_{2}^{\prime}} B-\mathbb{I}$ and $B$ are self-adjoint in $\mathbb{H}$, if functions $f_{1}$ and $f_{2}$ meet conditions 1) - 3) specified in the definition for operators $\mathbb{P}_{+}(v)$ and $\mathbb{P}_{-}(v)$. $1(y)$.

This property can be proved using item b) for the self-adjoint differential expression

\subsection{Eigenvalues and eigenfunctions of operator $\widehat{\mathbf{A}}$}

Theorem 1. The following multitudes

$$
\begin{gathered}
\left\{\lambda_{\mathrm{n}}=\mathrm{n}(\mathrm{n}+1)\right\}_{\mathrm{n}=0,}^{\infty}, \\
\left\{\varphi_{\mathrm{n}}=\mathrm{P}_{\mathrm{n}}\left(\mathrm{f}_{2}\right)\right\}_{\mathrm{n}=0,}^{\infty},
\end{gathered}
$$

are sets of eigenvalues and corresponding eigenfunctions of operator $\widehat{A}$ which is operative in $\mathbb{H} ; \mathrm{P}_{\mathrm{n}}(*) \$$ is Legendre's polynomial.

Proof. The operator is known (see [1, c.180]):

$$
A:=-\left(1-x^{2}\right) \frac{d^{2}}{d x^{2}}+2 x \frac{d}{d x}
$$

is classic Legendre operator which is operative in $\mathbb{H}$, herewith, the following equations are true:

$$
\operatorname{AP}_{\mathrm{n}}(\mathrm{x})=\lambda_{\mathrm{n}} \mathrm{P}_{\mathrm{n}}(\mathrm{x}) \quad \forall \mathrm{x} \in[-1,1]
$$


It means that $\left\{\lambda_{n}=n(n+1)\right\}_{n=0}^{\infty}$ and $\left\{\varphi_{n}=P_{n}\left(f_{2}\right)\right\}_{n=0}^{\infty}$ are sets of eigenvalues and corresponding eigenfunctions of the operator $\mathrm{A}$.

Based on requirements 1)-3) for functions $f_{1}(x)$ and $f_{2}(x)$ it appears that

$$
\begin{gathered}
f_{1}(x)=\frac{1-f_{2}^{2}(x)}{f_{2}^{\prime}(x)} \\
f_{1}^{\prime}(x)=-2 f_{2}(x)-\left(1-f_{2}^{2}(x)\right) \frac{f_{2}^{\prime \prime}(x)}{\left[f_{2}^{\prime}(x)\right]^{2}}
\end{gathered}
$$

Then

$$
\widehat{A}=-\frac{1-f_{2}^{2}(x)}{f_{2}^{\prime}(x)} \frac{d^{2}}{d x^{2}}+\left(\frac{2 f_{2}(x)}{f_{2}^{\prime}(x)}+\frac{\left(1-f_{2}^{2}(x)\right) f_{2}^{\prime \prime}(x)}{\left[f_{2}^{\prime}(x)\right]^{2}}\right) \frac{d}{d x}
$$

Therefore:

$$
\begin{gathered}
\widehat{A} P_{n}\left(f_{2}\right)=-\frac{1-f_{2}^{2}}{\left[f_{2}^{\prime}\right]^{2}}\left(P_{n}^{\prime \prime}\left(f_{2}\right)\left(f_{2}^{\prime}\right)^{2}+P_{n}^{\prime}\left(f_{2}\right) f_{2}^{\prime \prime}\right)+ \\
\frac{2 f_{2}}{f_{2}^{\prime}} P_{n}^{\prime}\left(f_{2}\right) f_{2}^{\prime}+\frac{\left(1-f_{2}^{2}\right) f_{2}^{\prime \prime}}{\left[f_{2}^{\prime}\right]^{3}} P_{n}^{\prime}\left(f_{2}\right) f_{2}^{\prime}=-\left(1-f_{2}^{2}\right) P_{n}^{\prime \prime}\left(f_{2}\right)+2 f_{2} P_{n}^{\prime}\left(f_{2}\right), \\
f_{1}=f_{1}(x), f_{2}=f_{2}(x) .
\end{gathered}
$$

Meaning that:

$$
\widehat{A} P_{n}\left(f_{2}(x)\right)=-\left(1-f_{2}^{2}(x)\right) P_{n}^{\prime \prime}\left(f_{2}(x)\right)+2 f_{2}(x) P_{n}^{\prime}\left(f_{2}(x)\right)
$$

And since

$$
\widehat{\mathrm{A}} \mathrm{P}_{\mathrm{n}}(\mathrm{x})=-\left(1-\mathrm{x}^{2}\right) \mathrm{P}_{\mathrm{n}}^{\prime \prime}(\mathrm{x})+2 \mathrm{x} \mathrm{P}_{\mathrm{n}}^{\prime}(\mathrm{x})
$$

and

$$
\mathrm{AP}_{\mathrm{n}}(\mathrm{x})=\lambda_{\mathrm{n}} \mathrm{P}_{\mathrm{n}}(\mathrm{x}) \quad \forall \mathrm{x} \in[-1,1]
$$

then, taking into account (13), we get

$$
\operatorname{AP}_{\mathrm{n}}\left(\mathrm{f}_{2}(\mathrm{x})\right)=\lambda_{\mathrm{n}} \mathrm{P}_{\mathrm{n}}\left(\mathrm{f}_{2}(\mathrm{x})\right) \quad \forall \mathrm{x} \in[-1,1]
$$

for function $\mathrm{f}_{2}(\mathrm{x})$, which meet the requirements 1)-3). The theorem is proved.

\subsection{The differentiation formula}

We know (see [1, c.180]) the following differentiation formula:

$$
\left(1-x^{2}\right) \frac{d P_{n}(x)}{d x}=n\left[P_{n-1}(x)-x P_{n}(x)\right]=(n+1)\left[x P_{n}(x)-P_{n+1}(x)\right] .
$$

Then

$$
\mathrm{P}_{\mathrm{n}+1}(\mathrm{x})=\mathbb{D}_{+}(\mathrm{n}) \mathrm{P}_{\mathrm{n}}(\mathrm{x}), \mathrm{P}_{\mathrm{n}-1}(\mathrm{x})=\mathbb{D}_{-}(\mathrm{n}) \mathrm{P}_{\mathrm{n}}(\mathrm{x})
$$

where 


$$
\begin{gathered}
\mathbb{D}_{+}(\mathrm{n}):=-\frac{\left(1-\mathrm{x}^{2}\right)}{(\mathrm{n}+1)} \frac{\mathrm{d}}{\mathrm{dx}}+\mathrm{xI}, \mathrm{n} \in \mathbb{N} \cup\{0\}, \\
\mathbb{D}_{\text {-(n) }}:=-\frac{\left(1-\mathrm{x}^{2}\right)}{\mathrm{n}} \frac{\mathrm{d}}{\mathrm{dx}}+\mathrm{xI}, \mathrm{n} \in \mathbb{N} .
\end{gathered}
$$

In items (15)-(17) we will replace $\mathrm{x}$ with $\mathrm{f}_{2}(\mathrm{x})$. Then $\frac{\mathrm{d}}{\mathrm{dx}}$ will be replaced with $\frac{1}{\mathrm{f}_{2}^{\prime}(\mathrm{x})} \frac{\mathrm{d}}{\mathrm{dx}}$, and oprators $\mathbb{D}_{+}(\mathrm{n}), \mathbb{D}_{-}(\mathrm{n})$ will be replaced with operators $\mathbb{P}_{+}(\mathrm{n}), \mathbb{P}_{-}(\mathrm{n})$. It follows from the above that:

Statement 3.

$$
\begin{gathered}
P_{n+1}\left(f_{2}\right)=P_{+}(n) P_{n}\left(f_{2}\right), \\
P_{n-1}\left(f_{2}\right)=P_{-}(n) P_{n}\left(f_{2}\right), n \in \mathbb{N} \cup\{0\},
\end{gathered}
$$

are the formulas for recurrent calculation of eigenfunctions $P_{n}\left(f_{2}\right)$ of the operator $\widehat{A}$.

\section{Examples of Legendre type operator acting in $\mathbb{H}$}

Let us consider several particular examples for the Legendre type operators which are operative in SHS $\mathbb{H}=\mathrm{L}_{2}(-1,1)$. Since the type and properties of operators are significantly dependent on the type and properties of functions $f_{1}$ and $f_{2}$ which may be of an unlimited choice (as long as the requirements 1)-3) are met for them), the number of Legendre type operators is quite vast. Eigenvalues and eigenfunctions for these operators may be found using formulas (11) and (12), accordingly.

Example 1.

$$
\widehat{A}=A:=-\left(1-x^{2}\right) \frac{d^{2}}{d x^{2}}+2 x \frac{d}{d x}
$$

is a classic Legendre operator. Here $\mathrm{f}_{2}(\mathrm{x})=\mathrm{x}$, then $\mathrm{f}_{1}(\mathrm{x})=1-\mathrm{x}^{2}$.

Example 2. Let us assume that $\mathrm{f}_{2}(\mathrm{x})=(\beta+\mathrm{x})^{\alpha}, \beta>1, \alpha \in \mathbb{R} \backslash\{0\}$. Then

$$
f_{1}(x)=-\frac{(\beta+x)\left(-(\beta+x)^{-\alpha}+(\beta+x)^{\alpha}\right)}{\alpha} \text {. }
$$

The operator $\widehat{\mathrm{A}}$ will become as follows:

$$
\widehat{A}:=-\frac{(\beta+x)^{2}\left((\beta+x)^{-2 \alpha}-1\right)}{\alpha^{2}} \frac{d^{2}}{d x^{2}}-\frac{\left((\beta+x)^{-2 \alpha+1}(1-\alpha)-\beta-x-\beta \alpha-x \alpha\right)}{\alpha^{2}} \frac{d}{d x} .
$$

Its eigenfunctions are: $P_{n}\left((\beta+x)^{\alpha}\right), n \in \mathbb{N} \cup\{0\}$.

Example 3. Let us assume that $f_{2}(x)=\ln (2+x)$. Then $f_{1}(x)=\left(1-\ln ^{2}(2+x)\right)(2+x)$. The operator $\widehat{\mathrm{A}}$ will become as follows:

$$
\widehat{A}:=\left(-1+\ln ^{2}(2+x)\right)(2+x)^{2} \frac{d^{2}}{d x^{2}}+\left(2 \ln (2+x)-1+\ln ^{2}(2+x)\right)(2+x) \frac{d}{d x} .
$$

Its eigenfunctions are: $\mathrm{P}_{\mathrm{n}}(\ln (2+\mathrm{x})), \mathrm{n} \in \mathbb{N} U\{0\}$.

\section{On homeomorphism of spaces}

We will prove the following lemma. 
Lemma 1. For every interval $[a, b](a<b)$ of finite length and every continuously differentiable function $\widetilde{\mathrm{f}_{2}}(\mathrm{x})$ with a derivative of fixed sign on this interval, there exist $\alpha$ and $\beta$ such that every monotone, continuously differentiable function $f_{2}(x)=\alpha \widetilde{f}_{2}(x)+\beta$ on [a,b] will have the following values: $f_{2}(a)=-1, f_{2}(b)=1$.

Proof. Let $\widetilde{\mathrm{f}_{2}}(\mathrm{a})=\mathrm{c}, \widetilde{\mathrm{f}_{2}}(\mathrm{~b})=\mathrm{d}$, then $\mathrm{c} \neq \mathrm{d}$ and we have to solve a linear system

$$
\left\{\begin{array}{c}
-1=\alpha c+\beta \\
1=\alpha d+\beta
\end{array}\right.
$$

where $\alpha$ and $\beta: \alpha=2 /(d-c), \beta=1-2 d /(d-c)$. This concludes the proof of the Lemma.

Theorem 2. Let $[a, b]$ be a random interval of finite length $(a<b)$ and $\widetilde{f_{2}}(x)$ is any continuously differentiable function with a derivative of fixed sign on this interval. Next we have $\widetilde{\mathrm{f}_{2}}(\mathrm{a})=\mathrm{c}, \widetilde{\mathrm{f}_{2}}(\mathrm{~b})=\mathrm{d}, \alpha=2 /(\mathrm{d}-\mathrm{c})$. Then the spaces $\mathbb{H}^{\omega, \mathrm{a}, \mathrm{b}}, \omega=\alpha \tilde{\mathrm{f}}_{2}^{\prime}(\mathrm{x})$, and $\mathbb{H}$ are homeomorphic.

Proof. In a Hilbert space $\mathbb{H}$ the functions $\varphi_{\mathrm{n}}=\frac{\mathrm{P}_{\mathrm{n}}}{\left\|\mathrm{P}_{\mathrm{n}}\right\|_{\mathrm{L}_{2}}}, \mathrm{n} \in \mathbb{N} U\{0\}$, form an orthonormal basis. According to the lemma, there exists $\beta$, such that the function $f_{2}(x)=\alpha \widetilde{f_{2}}(x)+\beta$ have the values: $f_{2}(a)=-1$ and $f_{2}(b)=1$ at the ends of the interval $[a, b]$.

Let's take the function $f(x) \in \mathbb{H}$. Then $g(t)=f\left(f_{2}(t)\right) \in \mathbb{H} \omega, a, b$ :

$$
\int_{a}^{b}\left|f\left(f_{2}(t)\right)\right|^{2} f_{2}^{\prime}(t) d t=\alpha \int_{a}^{b}\left|f\left(f_{2}(t)\right)\right|^{2} \tilde{f}_{2}^{\prime}(t) d t=\int_{-1}^{1}|f(x)|^{2} d x<\infty .
$$

Now let us consider the function $g(t) \in \mathbb{H}^{\omega, a, b}$. We can see from the properties of $\mathrm{f}_{2}$ that $t=f_{2}{ }^{-1}(y)$ is a monotone, continuously differentiable function on $[-1,1]$.

It is obvious that $\mathrm{f}(\mathrm{y})=\mathrm{g}\left(\mathrm{f}_{2}{ }^{-1}(\mathrm{y})\right) \in \mathbb{N}$ :

$$
\begin{gathered}
\int_{-1}^{1}|f(y)|^{2} d y=\int_{-1}^{1}\left|g\left(f_{2}^{-1}(y)\right)\right|^{2} d y= \\
\int_{a}^{b}|g(t)|^{2} f_{2}^{\prime}(t) d t=\alpha \int_{a}^{b}|g(t)|^{2} \tilde{f}_{2}^{\prime}(t) d t<\infty .
\end{gathered}
$$

We can see that the spaces $\mathbb{H}$ and $\mathbb{H} \mathbb{H}^{\omega, \mathrm{a}, \mathrm{b}}$ are isomorphic. Considering

$$
\int_{a}^{b} P_{n}\left(f_{2}(x)\right) P_{m}\left(f_{2}(x)\right) f_{2}^{\prime}(x) d x=\int_{-1}^{1} P_{n}(u) P_{m}(u) d u=\delta_{n m}
$$

we get that

$$
\psi_{\mathrm{n}}=\frac{\mathrm{P}_{\mathrm{n}}\left(\mathrm{f}_{2}(\mathrm{t})\right)}{\left\|\mathrm{P}_{\mathrm{n}}\left(\mathrm{f}_{2}(\mathrm{t})\right)\right\|_{\mathrm{L}_{2} \omega}}, \mathrm{n} \in \mathbb{N} U\{0\}
$$

is an orthonormal basis in $\mathbb{H}^{\omega, \mathrm{a}, \mathrm{b}} ; \delta_{\mathrm{nm}}$ it is the symbol of Kronecker.

It is then easy to verify usingthe Lebesgue integral properties that

$$
\mathrm{G}(\mathrm{f}): \mathbb{H} \ni \mathrm{f}(\mathrm{x}) \mapsto \mathrm{f}\left(\mathrm{f}_{2}(\mathrm{t})\right) \in \mathbb{H}^{\omega, \mathrm{a}, \mathrm{b}}
$$

it is not only bijective relationship of spaces, but also continuous together with its inverse relation. This is possible if the elements considered spaces to take classes of functions that differ from each other on the set of measure zero.

Let functions $f_{n}$ are a Cauchy sequence in $\mathbb{H}$ and $f \in \mathbb{H}$, with $\left\|f_{n}-f\right\| \rightarrow 0, n \rightarrow \infty$, then (18): $\left\|g_{n}-g\right\|_{\omega} \rightarrow 0$, where $g_{n}(t)=f_{n}\left(f_{2}(t)\right), g(t)=f\left(f_{2}(t)\right)$. 
If the functions $\mathrm{g}_{\mathrm{n}}$ are a Cauchy sequence in $\mathbb{H}^{\omega, \mathrm{a}, \mathrm{b}}$ and $\mathrm{g} \in \mathbb{H}^{\omega, \mathrm{a}, \mathrm{b}}$, with $):\left\|\mathrm{g}_{\mathrm{n}}-\mathrm{g}\right\|_{\omega} \rightarrow 0$, $\mathrm{n} \rightarrow \infty$, then (19): $\left\|\mathrm{f}_{\mathrm{n}}-\mathrm{f}\right\| \rightarrow 0$, where $\mathrm{f}_{\mathrm{n}}(\mathrm{y})=\mathrm{g}_{\mathrm{n}}\left(\mathrm{f}_{2}{ }^{-1}(\mathrm{y})\right), \mathrm{f}(\mathrm{y})=\mathrm{g}\left(\mathrm{f}_{2}{ }^{-1}(\mathrm{y})\right)$. Thus, $\mathrm{G}\left({ }^{*}\right)$ is a homeomorphism. This finishes the proof.

\section{Legendre operator $\widehat{\mathbf{A}}$ in weight space $\mathbb{H}^{\omega, a, b}$}

Let functions $\mathrm{f}_{1}=\mathrm{f}_{1}(\mathrm{t})$ and $\mathrm{f}_{2}=\mathrm{f}_{2}(\mathrm{t})$ on a random interval $[\mathrm{a}, \mathrm{b}](\mathrm{a}<\mathrm{b})$ of finite length have the following properties:

1) $f_{2}$ is a twice continuously differentiable function supported on [a,b];

2) equality $t \in[a, b]$ is true for any $f_{1}(t) f_{2}(t)+f_{2}{ }^{2}(t)=1$;

3) $f_{2}{ }^{\prime}$ is a function of fixed sign on $[a, b]$ and

4) $f_{2}(a)=-1, f_{2}(b)=1$.

It is easy to construct the function $\mathrm{f}_{2}$ using the lemma.

Let the operator

$$
\widehat{A}=-\frac{f_{1}}{f_{2}^{\prime}} \frac{d^{2}}{{d t^{2}}^{2}}-\frac{f_{1}^{\prime}}{f_{2}^{\prime}} \frac{d}{d t}
$$

acts in $\mathbb{H}^{\omega, a, b}, \omega=f_{2}^{\prime}(t)$.

The domain of definition of the operator $\widehat{A}$ includes all functions and their first derivatives that are absolutely continuous on the interval [a,b]. The second derivatives (functions must have a second derivative at each point of [a,b]) are with integrable square on this interval.

Let $\mathrm{f}(\mathrm{t}) \in \mathrm{D}(\widehat{\mathrm{A}}) \subset \mathbb{H}^{\omega, \mathrm{a}, \mathrm{b}}$. Then $\mathrm{f}\left(\mathrm{f}_{2}^{-1}(\mathrm{x})\right)=\mathrm{g}(\mathrm{x}) \in \mathrm{D}(\mathrm{A}) \subset \mathbb{H}$ :

$$
\begin{gathered}
\infty>\int_{a}^{b}|f(t)|^{2} f_{2}^{\prime}(t) d t=\int_{-1}^{1}\left|f\left(f_{2}^{-1}(x)\right)\right|^{2} d x . \\
A:=-\left(1-x^{2}\right) \frac{d^{2}}{d x^{2}}+2 x \frac{d}{d x}
\end{gathered}
$$

is the Legendre operator (see, for example, [1]), acting in $\mathbb{H}$ (note that $A=\widehat{A}$, if $f_{2}(x)=x$, a $=-1, b=1)$. Considering the equality $\mathrm{f}_{1}(t) \mathrm{f}_{2}{ }^{\prime}(\mathrm{t})+\mathrm{f}_{2}{ }^{2}(\mathrm{t})=1(\mathrm{t} \in[\mathrm{a}, \mathrm{b}])$ and the function $\mathrm{x}=\mathrm{f}_{2}(\mathrm{t})$ $(t \in[a, b], x \in[-1,1])$, we get that

$$
\begin{gathered}
\operatorname{Af}\left(\mathrm{f}_{2}^{-1}(\mathrm{x})\right)=-\left(1-\mathrm{f}_{2}^{2}(\mathrm{t})\right)\left(\frac{\mathrm{f}^{\prime \prime}(\mathrm{t})}{\left(\mathrm{f}_{2}^{\prime}(\mathrm{t})\right)^{2}}-\frac{\mathrm{f}^{\prime}(\mathrm{t}) \mathrm{f}_{2}^{\prime \prime}(\mathrm{t})}{\left(\mathrm{f}_{2}^{\prime}(\mathrm{t})\right)^{3}}\right)+2 \mathrm{f}_{2}(\mathrm{t}) \frac{\mathrm{f}^{\prime}(\mathrm{t})}{\mathrm{f}_{2}^{\prime}(\mathrm{t})}=\cdots= \\
\left(-\frac{\mathrm{f}_{1}(\mathrm{t})}{\mathrm{f}_{2}^{\prime}(\mathrm{t})} \frac{\mathrm{d}^{2}}{d \mathrm{t}^{2}}-\frac{\mathrm{f}_{1}^{\prime}(\mathrm{t})}{\mathrm{f}_{2}^{\prime}(\mathrm{t})} \frac{\mathrm{d}}{\mathrm{dt}}\right) \mathrm{f}(\mathrm{t})=\widehat{A} \mathrm{f}(\mathrm{t}) .
\end{gathered}
$$

That is $\mathbb{H} \ni \operatorname{Af}\left(\mathrm{f}_{2}^{-1}(\mathrm{x})\right)=\widehat{\operatorname{Af}} \mathrm{f}(\mathrm{t}) \in \mathbb{H}^{\omega, \mathrm{a}, \mathrm{b}}, \omega=\mathrm{f}_{2}{ }^{\prime}(\mathrm{t}), \mathrm{t} \in[\mathrm{a}, \mathrm{b}], \mathrm{x}=\mathrm{f}_{2}(\mathrm{t}) \in[-1,1]$.

The action of the operator $\widehat{A}$ in $\mathbb{H}^{\omega, a, b}$ can be therefore replaced with the action of the operator $A$ in $\mathbb{H}$, only instead of $f(t) \in D(\widehat{A})) \subset \mathbb{H}^{\omega, a, b}$ there should be

$$
\mathrm{f}\left(\mathrm{f}_{2}{ }^{-1}(\mathrm{x})\right)=\mathrm{g}(\mathrm{x}) \in \mathrm{D}(\mathrm{A}) \subset \mathbb{H} .
$$

The result of the action $v(t)=\widehat{A} f(t)$ will be equal to $\operatorname{Ag}(x)=\omega(x)=\omega\left(f_{2}(t)\right)$, that is

$$
v(t)=\omega\left(f_{2}(t)\right)
$$


This completes the proof of the following theorem.

Theorem 3. Let the function $x=f_{2}(t)$ have the properties specified in items 1)-4). Then the equality $f(t) \in D(\widehat{A})) \subset \mathbb{H}^{\omega, a, b}$ is true for any function

$$
\mathbb{H} \ni \operatorname{Af}\left(\mathrm{f}_{2}^{-1}(\mathrm{x})\right)=\widehat{\mathrm{A}} \mathrm{f}(\mathrm{t}) \in \mathbb{H}^{\omega, \mathrm{a}, \mathrm{b}} .
$$

Here $\omega=f_{2}^{\prime}(t), t \in[a, b]$.

\section{Examples of Legendre type operator acting in $\mathbb{H}^{\omega, a, b}$}

In each of the following examples for the given function $f_{2}(t)$ we have found values of a and $b$ and the weigh $\omega$ such that the conditions of Theorem 1 are applied, i.e. the spaces $\mathbb{H}^{\omega, \mathrm{a}, \mathrm{b}}$ and $\mathbb{H}$ are homeomorphic.

Example 1. Let us consider the function $\widetilde{\mathrm{f}_{2}}(\mathrm{t})=\operatorname{ctg}\left(\mathrm{e}^{\mathrm{x}}\right)$. Then let $\mathrm{a}=\ln (\pi / 4), \mathrm{b}=$ $\ln (\pi / 2)$, as $\tilde{\mathrm{f}}_{2}^{\prime}(\mathrm{t})=-\frac{\mathrm{e}^{\mathrm{t}}}{\sin ^{2} \mathrm{e}^{\mathrm{t}}}<0$ on $[\mathrm{a}, \mathrm{b}]$. Using $\mathrm{c}=\widetilde{\mathrm{f}_{2}}(\mathrm{a})=\operatorname{ctg}(\exp (\ln (\pi / 4)))=1, \mathrm{~d}=\widetilde{\mathrm{f}_{2}}(\mathrm{~b})$ $=\operatorname{ctg}(\exp (\ln (\pi / 2)))=0$ by the lemma, we will find $\alpha$ and $\beta: \alpha=-2, \beta=1$. It therefore follows that $f_{2}(t)=-2 \operatorname{ctg}\left(e^{t}+1\right)$, and the weight is

$$
\omega=\mathrm{f}_{2}{ }^{\prime}(\mathrm{t})=2 \frac{\mathrm{e}^{\mathrm{t}}}{\sin ^{2} \mathrm{e}^{\mathrm{t}}} .
$$
that

Example 2. Let us consider the function $\widetilde{\mathrm{f}_{2}}(\mathrm{t})=\log _{2}\left(1+\mathrm{t}^{2}\right)$. Let $\mathrm{a}=0, \mathrm{~b}=1$. Considering

$$
\begin{gathered}
c=\widetilde{\mathrm{f}_{2}}(\mathrm{a})=0, \mathrm{~d}=\widetilde{\mathrm{f}_{2}}(\mathrm{~b})=1, \\
\alpha=2, \beta=-1 .
\end{gathered}
$$

As a result, we have $f_{2}(t)=2 \log _{2}\left(1+t^{2}\right)-1$, and the weight

$$
\omega=4 \mathrm{t} /\left(\left(1+\mathrm{t}^{2}\right) \ln 2\right) \text {. }
$$

Example 3. Let us consider the function $\widetilde{\mathrm{f}_{2}}(\mathrm{t})=\arctan \sqrt{\mathrm{t}}, \mathrm{t} \geq 0$. Then let $\mathrm{a}=1, \mathrm{~b}=3$. Considering

$$
\begin{gathered}
c=\widetilde{f_{2}}(a)=\frac{\pi}{4}, d=\widetilde{f_{2}}(b)=\frac{\pi}{3}, \\
\alpha=24 / \pi, \beta=-7 .
\end{gathered}
$$

In the result, we have $f_{2}(t)=(24 / \pi) \arctan \sqrt{t}-7$, and the weight

$$
\omega=\frac{12}{\pi \sqrt{\mathrm{t}}(1+\mathrm{t})} \text {. }
$$

Remark. Having calculated $\mathrm{f}_{1}(\mathrm{t})$ using the formula $\mathrm{f}_{1}(\mathrm{t})=\left(1-\mathrm{f}^{2}{ }_{2}(\mathrm{t})\right) / \mathrm{f}^{\prime}(\mathrm{t})$, in examples 1 3 , we can explicitly present the operator $\widehat{A}$, for which functions $P_{n}\left(f_{2}(t)\right)$ are eigenfunctions corresponding to the eigenvalues $\lambda_{n}=n(n+1), n \in \mathbb{N} U\{0\}$.

\section{Conclusions}

The article presents a new class of differential operators. It is obtained through a generalization of the classical Legendre type operators. The action of operators from this 
class is best viewed in separable weighted Hilbert spaces. I think that Legendre type operators can be well used in mathematical physics.

If we substitute the basic elementary functions instead of the function $\mathrm{f}_{2}(\mathrm{x})$, then we can obtain a classification of Legendre type operators. For example, if we take a polynomial instead of $f_{2}(x)$, then expressing the function $f_{1}(x)$ from the equation $f_{1}(t) f_{2}{ }^{\prime}(t)+f_{2}{ }^{2}(t)=1$, we obtain a Legendre type operator with coefficients in the form of rational functions. And so on. The selection of the corresponding weighted Hilbert space is described in detail in paragraphs 6 and 7.

\section{References}

1. G. Bateman, A. Erday, Science, 296 (1974)

2. M.A. Naimark, Linear differential operators, 352 (1954)

3. E.M. Maleko, I.A. Maximenko, Scientific Herald of the Voronezh state University, 2, 249 (2012) 